\title{
The world status of the Houbara Bustard Chlamydotis undulata
}

\author{
PAUL D. GORIUP
}

\section{Summary}

The Houbara Bustard Chlamydotis undulata is prominent in avian conservation because of its high value as the traditional quarry of falconers in Arabia and western Asia; such hunting has often been blamed for severely reducing population levels especially in Arabia, Pakistan and Morocco. This paper reviews current knowledge of the world status of the Houbara Bustard in each of the 31 range states where it has occurred within the last 10 years. On the basis of the evidence available, the overall population is estimated to be between 49,000 and 62,000 birds, made up of $700-750$ birds in the Canary Islands, around 10,000 in Africa, and the remainder in the Middle East and Asia (especially Iran, Kazakhstan, Uzbekistan and Turkmenistan). Numbers have apparently declined greatly during this century because of agricultural intensification and other land use changes, often exacerbated by hunting and trapping. Evaluation of the population status and trends against the current Red List criteria of IUCN indicate that the Canary Islands subspecies C. $u$. fuertaventurae is Vulnerable, while the subspecies C. u. undulata and C. $u$. macqueenii (as well as the whole species population) could probably also qualify for listing as Vulnerable but it is more appropriate to consider them "Near threatened" for the time being.

\section{Introduction}

The Houbara Bustard Chlamydotis undulata is a medium-sized member of the family Otididae that occurs across the arid belt from the Canary Islands (Spain), North Africa and the Middle East to central and eastern Asia (Figure 1). It inhabits fairly level plains with gentle slopes used for display and nesting (Osborne 1996). The vegetation of the plains may range from absent to sub-Mediterranean, Indo-Saharan, Turanian, Central Asian and Mongolian semi-desert grassland, Artemisia shrubland and wooded steppe, provided cover density does not exceed about $25 \%$ and average height about $0.4 \mathrm{~m}$. It is resident, dispersive and migratory, the degree and regularity of movements tending to become more pronounced toward the north and east of its range. In general, the Houbara Bustard is sparsely distributed, but can occur in some numbers on migration and in favourable wintering areas (Cramp and Simmons 1980, Osborne 1996).

The Houbara Bustard has a high profile among conservationists because it is highly valued as the traditional quarry of Arab falconers (Goriup 1985, Osborne 1996, Upton 1989). Originally sought as a valuable additional food source in the winter, the practice of hunting the Houbara Bustard has now become a sport 


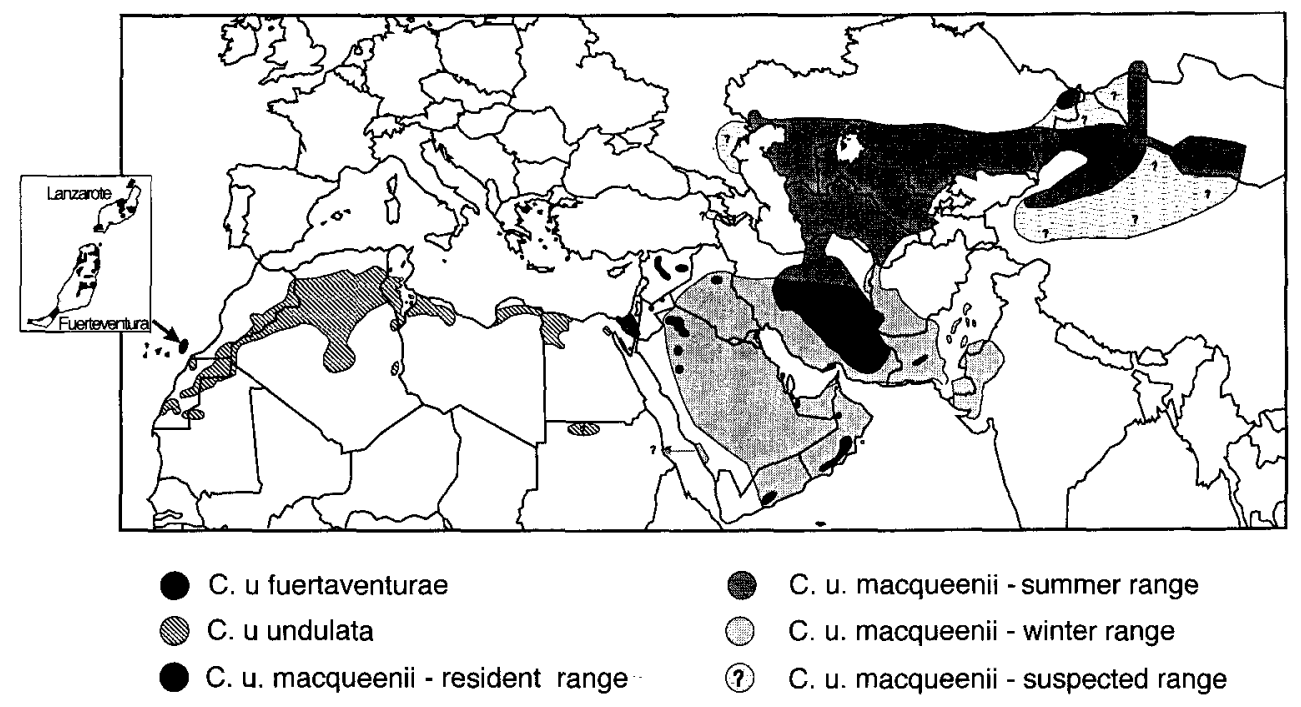

Figure 1. World distribution of the Houbara Bustard Chlamydotis undulata based on the sources given in this paper. The distribution is better known in Morocco, Tunisia, Egypt, Israel, the Arabian peninsula, Pakistan, and India than elsewhere.

intensively pursued by hundreds of people, including members of ruling families, in many Gulf States as well as North Africa, Pakistan and Central Asia.

The late 1960s saw the advent of "mechanized" falconry, employing fleets of all-terrain vehicles, sophisticated tracking and communications equipment, dozens of falcons and increasing use of firearms; parties began to roam through North Africa, the Sahel, Middle East and western Asia in search of their trophies, sometimes gaining access to hunting grounds through oil-based coercion and bribery (Newby 1990, Weaver 1992). The resulting indiscriminate and very often illegal hunting of Houbara Bustards (as well as other bustards, gazelles and antelopes that the hunting parties encountered), especially in Pakistan and Morocco, caused considerable concern among conservationists (Roberts and Savage 1971) and was one of the factors which led to the establishment of the then ICBP Bustard Specialist Group (BSG) in 1972. One of the original aims of the BSG was to devise captive breeding schemes, based on European experience with the Great Bustard Otis tarda, in the hope of setting up "put and take" systems for the Houbara Bustard to reduce pressure on wild populations. The BSG grew rapidly (and still exists under the joint supervision of the IUCN Species Survival Commission and BirdLife International), but its emphasis has shifted from captive breeding to dealing with the sustainable management of wild populations of bustards and their habitats as this appears to be more appropriate.

The last, admittedly preliminary, global review of the status of the Houbara Bustard was carried out by Collar ( 1980 ). He covered 18 countries, but one of these was the former U.S.S.R. which now comprises six individual range states of the Houbara Bustard, with the bulk of the population in Kazakhstan, Uzbekistan and Turkmenistan rather than Russia. In 15 of these countries, Collar 
(1980) reported evidence of decline over the past century, attributed to excessive hunting, overgrazing of habitat, agricultural development, industrial and urban development, and taking eggs for food. Based on these and other findings, the Houbara Bustard was classified as Vulnerable in the 1993 IUCN Red List of Threatened Animals (Groombridge 1993). However, as a result of introducing new criteria for evaluating the threatened status of species based on population dynamics and biogeography, the Houbara Bustard was not included as a threatened species in Birds to watch 2 (Collar et al. 1994). After Collar et al. (1994) was published, the criteria for some of the IUCN Red List Categories were amended. Thus while the 1996 IUCN Red List of Threatened Animals (IUCN 1996), for the sake of consistency with Collar et al. (1994), still does not consider the Houbara Bustard threatened, this review suggests that according to the amended criteria, at least $C$. $u$. fuertaventurae should be considered as Vulnerable, if not the species as a whole.

\section{Range and status}

\section{C. u. undulata}

This subspecies, the African Houbara Bustard, is sedentary and dispersive, wandering in search of fresh feeding areas (especially where rain has recently fallen). It occurs in the following eight range states.

Algeria Collar (1980) showed that the Houbara Bustard had been generally common in the latter part of the nineteenth century and early part of this century (e.g. Rothschild and Hartert 1912); however, numbers had started to decline by the 1920 as more arid areas were brought into cultivation and oil exploration took place (Heim de Balzac 1924; A. R. Dupuy verbally to N. J. Collar, 1979). According to de Smet (1989) it is a breeding resident which has declined locally since the 1960s (especially in the high plains of the Atlas range), but still occurs in some numbers in the pre-desert zone of the Auree mountains and Saharan Atlas. These areas are subject to occasional hunting by falconers from Saudi Arabia, who can kill over 1,00o birds in a season (de Smet 1989, pers. obs. 1988). In 1986 and 1987, eggs and chicks were collected from an area $80 \mathrm{~km}$ south-east of Abiod Sidi le Sheikh for a breeding scheme at Taif in Saudi Arabia (Gaucher 1988,1991 ); in 1987 alone 56 nests (holding a total of 130 eggs) were found during 28 days of searching and 22 of these nests came from an area of only 6,300 ha, indicating that relatively high breeding densities could still be found in the country.

Egypt Collar (1980) found recent information to be very feeble. According to Meinertzhagen (1930), the Houbara Bustard was not uncommon round the Wadi Natrun at all seasons, and observed between Siwa and Salum in February 1920, and at Kharga and Dakhla in spring. Bulman (1942) found the birds only in the hinterland of Matruh, in a strip 16 to $40 \mathrm{~km}$ from the coast and 150 to $250 \mathrm{~m}$ above sea-level. Collar (1980) also received information from R. Upton that an Arab falconry party had killed several hundred birds during a visit in the mid-1970s. The Houbara Bustard is now regarded as a breeding resident in the 
far western part of the Western Desert about $50 \mathrm{~km}$ inland from the coast and along the border with Algeria (Goodman and Meininger 1989, Saleh 1989). As mentioned previously, it was formerly more widespread, especially in the Wadi el Natrun west of the Nile delta, and in some southern localities, but seems to have disappeared from these during the 1950 s and 1960s. Shooting by local hunters still takes place in the Western Desert, along the Mediterranean coast and around the Siwa Oasis where bags of 20 to 30 birds per day are reported. Other causes of decline include overgrazing, agricultural development, unregulated vehicle use and collection of fuelwood that destroys native vegetation (Egyptian National Report to CMS, 1994).

Libya Breeding resident but current status unknown. It seems to have been fairly common up to the 1940 shen flocks of 50 and even 100 birds were reported (Bulman 1942). However, Bundy (1976) gave no recent records of such large flocks, and although he thought it probably still bred across northern Libya, he stated that it was subject to severe shooting pressure. Various contemporary sources suggest that such local hunting still continues, but visits by falconers from Gulf countries are very infrequent.

Mauritania Breeding resident in the north of the country on the borders with Western Sahara, Morocco and Algeria, but current status unknown (Morel 1989, Urban et al. 1986).

Morocco Collar (1980) noted that the Houbara Bustard in Morocco had received the most attention in North Africa, in particular from Brosset (1961). At that time, it was relatively common in remoter areas that could not be reached by hunters in cars, but from the 1970s hunting pressure increased as Arab falconers began to visit the country ( $P$. Glasier and R. Upton, verbally to N. J. Collar, 1979). It is a breeding resident with a wide distribution in the east and south of the country, including some plains and wider valleys of the Atlas range, but generally scarce (Goriup 1983). Most of the flatter pre-Saharan areas have been allocated to falconers from Saudi Arabia, U.A.E. and Bahrain over the past 25 years, where bags of several hundred birds per year are reported and there have been severe declines of the populations subject to hunting (Brahim Haddane, verbally 1995). Captive breeding and release stations have been financed by falconers from Saudi Arabia (completed in 1993) and U.A.E. (under construction) at the suggestion of King Hassan (Brahim Haddane, verbally 1995).

Sudan Apparently occurs in the north of the country but if so current status unknown (Urban et al. 1986). Hunting parties from Saudi Arabia regularly visit Sudan and thousands of bustards have been killed (Cloudsley-Thompson 1992), but whether these were Houbara Bustards or some other species is not known. An old record of a Houbara Bustard at Port Sudan may indicate vagrancy of $C$. u. macqueenii across the Red Sea.

Tunisia Collar (1980) reviewed the scant literature from the first half of this century, which contained evidence of declines (e.g. Bédé 1928), but that it was still quite common in southern regions at least until the mid-1930s (Blanchet 
1955). A breeding resident in suitable areas in the centre and southern parts of the country (Anon. 1980). Flocks of 10 or so birds have been seen in the Kasserine region. Hunting by falconers from Saudi Arabia persists (A. el Hili, verbally 1994).

Western Sahara Breeding resident occurring in the north-east part of the territory (Valverde 1957, Brahim Haddane, verbally 1995) on the borders with Morocco, Mauritania and Algeria. Its status is unknown, however.

\section{C. u. fuertaventurae}

This subspecies, the Canarian Houbara Bustard, is restricted to the islands of Fuerteventura and Lanzarote with a few birds also inhabiting the islet of Graciosa off Lanzarote in the eastern Canary Islands (Spain). There is some exchange between the islands (Martin et al. 1996) and the possibility of vagrancy between the archipelago and Morocco cannot be ruled out (the colonizers presumably originated from Morocco). Modern studies on C. u. fuertaventurae began only recently (e.g. Collar and Goriup 1983, Collins 1984, Osborne 1986, Hinz and Heiss 1989): it was considered possibly extinct by Bannerman (1963). However, this small population of $700-750$ birds has now become one of the best studied and managed of the whole species.

The most recent estimate of the population on Fuerteventura is $300-350$ birds in 1992 (ORNISTUDIO 1992). This compares with an estimate of $42-100$ in 1979 (Collar and Goriup 1983) and very few others on Lanzarote. On the latter though, census work in 1993 established that the population of Houbara Bustards on the island is much larger than hitherto realized, numbering at least 130 birds, with another 15 or more inhabiting the islet of Graciosa (Martin et al. 1996); the estimated total population for these two islands is about 400 birds. While some of this increase must result from better survey efficiency, it appears that the population has increased over the past 10 years, probably because of improved protection from poaching, reduction in grazing as a result of agricultural decline, and habitat management within the protected areas (e.g. irrigating patches of fodder plants).

The total extent of suitable habitat on "Fuerteventura has been estimated as about 34,300 ha, mainly in the north where two protected areas have been established, but with a significant block of 4,560 ha $(13 \%)$ covering the Matas Blancas (Jandia isthmus) of which a core area of 2,754 ( $8 \%$ of the total) has been designated as a Special Protection Area under the EC Birds Directive of 1979 . This is the maximum extent of habitat available and it is gradually eroding from overgrazing, road building and tourist development. The two islands hold some 17,500 ha of habitat (about half of that available on Fuerteventura), which is subject to deleterious impacts from off-road vehicles (tourist joy-riding), military exercises, and expanding infrastructure of roads and holiday complexes. There are no protected areas for the Houbara Bustard on Lanzarote.

A detailed recovery plan for the Canarian Houbara Bustard was drafted by Dominguez and Diaz (1985) but it is now in urgent need of updating and then full implementation. A more recent Action Plan has been produced by BirdLife International (Heredia et al. 1996) on behalf of the Council of Europe. The 
importance of this isolated yet very accessible population for research and experimental management should be stressed in any global conservation plan for the species. In fact, a research station was established at La Oliva in Fuerteventura ( $F$. Dominguez, verbally 1992) and captive breeding was attempted, but without success.

\section{C. u. macqueenii}

This subspecies, the Asian Houbara Bustard, is resident, dispersive and migratory, with a high incidence of vagrancy to Western Europe until the 1970s (Cramp and Simmons 1980). The degree and regularity of its movements become more pronounced toward the north and east of its range. Since 1995, there has been a satellite tracking programme undertaken by the National Avian Research Centre (NARC) from Abu Dhabi, U.A.E., and the Institute of Zoology at Almaty in Kazakhstan, that is yielding vital information about the pattern and degree of migratory behaviour (F. Launay, verbally 1995). In 1994, the IUCN Species Survival Commission established an Asian Houbara Bustard Working Group which held its first technical meeting in Muscat from 13 to 14 January 1996 in collaboration with the Ministry of Regional Municipalities and Environment, and the Secretariat of the Convention on Conservation of Migratory Species of Wild Animals (CMS).

The overall range of $C$. $u$. macqueenii may be conveniently divided into six subregions (modified somewhat from Osborne, 1996), although the actual degree of separation and interaction between the populations concerned is presently unknown:

(i) Middle East: comprising resident or locally migratory breeding birds in Syria, western Iraq, Jordan, Israel, Egypt (Sinai), northern Saudi Arabia and possibly the Aras valley in the Transcaucasus

(ii) Eastern Arabian Peninsula: comprising resident or locally migratory breeding birds in eastern Saudi Arabia, Oman and, formerly, south-east U.A.E.

(iii) Irano-Pakistan: comprising resident or locally migratory breeding birds in central Iran to western Baluchistan, Pakistan

(iv) Turanian: comprising birds that breed in western Kazakhstan, Uzbekistan and Turkmenistan and migrate to winter in Iran, Iraq and possibly the northern Arabian peninsula

(v) Central Asian: comprising birds that breed in central and eastern Kazakhstan, Uzbekistan and Turkmenistan and migrate to winter apparently in eastern Iran, Pakistan, north-west India and possibly the central and eastern Arabian Peninsula as far south as the Tihama plain of the Red Sea coast

(vi) Mongolian Plateau and Gobi Desert: comprising birds that breed in the extreme east of Kazakhstan, arid zone of southern Russia (Siberia), southern Mongolia and north-west China and migrate to winter (but precise migration routes and wintering grounds are unknown)

Excluding countries where it has occurred only as a vagrant, there are 23 range states of C. $u$. macqueenii. The inventory of important bird areas (IBAs) in the 
Middle East (Evans 1994) lists 60 sites where C. u. macqueenii has been reported, or may occur (Table 1, Figure 2). The initial results from the satellite tracking exercise (Figure 2) strongly suggest that there is indeed a "migration divide" between Houbara Bustard populations in western and eastern Kazakhstan (further evidence of this was obtained by NARC during 1996). Moreover, the fact that the broad routes followed, albeit based on only seven birds tracked, take in so many IBAs suggests that a closer analysis of the role of these sites will be vital in developing any future conservation programme for C. u. macqueenii.

Afghanistan Collar (1980) noted only that records are extremely scarce and that C. D. W. Savage (in litt. to N. J. Collar, 1979) had received information that they were not very numerous in winter. From the results of satellite tracking work by NARC in Kazakhstan (Figure 2), the Houbara Bustard is clearly a passage migrant and winter visitor, as one of the telemetered birds reached the sole IBA site known for the Houbara Bustard, situated in the Registan Desert; it may also breed in the country (Evans 1994). Census work in the Chagai region of Baluchistan carried out by WWF-Pakistan (Chaudhry 1996) provides evidence that many hundreds of birds may spend some time in Afghanistan before moving south, and moreover falconers from Saudi Arabia and Dubai (UAE) hunted in the Registan Desert in 1995 and 1996 under protection from local chiefs (Brig. Mukhtar Ahmed, verbally 1996). A local NGO, Society for Afghanistan Volunteer Environmentalists, is planning to undertake survey work to gain a better understanding of the present situation (Adil 1996).

Azerbaijan There are unconfirmed reports of recent occurrences from hunters and game wardens, for example along the foot of the Negram Mountains in Nakhichevan region (M. Patrikeev, in litt. 1996).

Armenia Reported by Prof. Ednusrd Yavrouyan as a passage migrant and wintering bird in the south of the country, particularly in the Aras (Araxes) valley (Armenia National Report to CMS, 1994). In addition, there may be occasional breeding in protected areas such as at Khosrov Reserve. Once fairly common, after the 1950 s numbers declined rapidly and the Houbara Bustard is now extremely rare. Much of the decline was due to increased urbanization, as well as hunting and, in recent years, intense military activity.

Bahrain Formerly said to breed (Belgrave 1953) but now a rare migrant and winter visitor in ones or twos from October to March from Sar south to Ra's al Barr, with records from Al Areen Wildlife Park and Bahrain airport (April 1992). Visiting birds are usually hunted, with several tens killed each year (up to 60 reported in 1988), but numbers have greatly diminished over the past 50 years (Nightingale and Hill 1993, Hirschfeld 1995). A captive breeding scheme was established at Al Areen Wildlife Park in 1976, but so far no progeny have been produced (Bahrain National Report to CMS, 1995).

China Resident, and passage migrant (presumably from Mongolia), confined to suitable plains in Xinjiang Province only. Wintering grounds unknown and overall status unclear (Gao Xing-Yi, in litt. 1995). The most recently published 
Table 1. Important Bird Areas in the Middle East where C. u. macqueenii has been observed or occurrence is suspected. The sites are plotted in Figure 2

\begin{tabular}{|c|c|c|c|c|}
\hline Country & Site name & Location & $\begin{array}{l}\text { Area } \\
\left(\mathrm{km}^{2}\right)\end{array}$ & Notes \\
\hline Afghanistan & $\begin{array}{l}\text { North-western steppe, } \\
\text { Herat and Badgis }\end{array}$ & $35^{\circ} 10^{\prime} \mathrm{N} 62^{\circ} \mathrm{oo}{ }^{\prime} \mathrm{E}$ & 800 & $\begin{array}{l}\text { Possibly breeds; one bird } \\
\text { present in July } 1949\end{array}$ \\
\hline Afghanistan & Registan Desert, Kandahar & $30^{\circ} 30^{\prime} \mathrm{N} 65^{\circ} 00^{\prime} \mathrm{E}$ & 30,000 & Possibly breeds \\
\hline Iran & $\begin{array}{l}\text { Miankaleh Peninsula and } \\
\text { Gorgan Bay, } \\
\text { Mazandaran }\end{array}$ & $36^{\circ} 50^{\prime} \mathrm{N} 53^{\circ} 45^{\prime} \mathrm{E}$ & 972 & Two records of migrants \\
\hline Iran & Kavir Region, Tehran & $34^{\circ} 45^{\prime} \mathrm{N} 52^{\circ} 10^{\prime} \mathrm{E}$ & 6,700 & $\begin{array}{l}\text { Summer visitor; } \\
\text { maximum count } 6 \text { birds }\end{array}$ \\
\hline Iran & $\begin{array}{l}\text { Khosh-Yeilagh, } \\
\text { Mazandaran }\end{array}$ & $36^{\circ} 50^{\prime} \mathrm{N} 55^{\circ} 35^{\prime} \mathrm{E}$ & 1,544 & $\begin{array}{l}\text { Reported to occur on } \\
\text { passage }\end{array}$ \\
\hline Iran & Touran, Semnan & $35^{\circ} 40^{\prime} \mathrm{N} 56^{\circ} 20^{\prime} \mathrm{E}$ & 18,727 & $\begin{array}{l}\text { Relatively common } \\
\text { summer visitor, May- } \\
\text { November; maximum } \\
18 \text { birds. }\end{array}$ \\
\hline Iran & $\begin{array}{l}\text { Tayebad Plains at Ghoomi } \\
\text { and Sarhad, Khorasan }\end{array}$ & $34^{\circ} 45^{\prime} \mathrm{N} 60^{\circ} 55^{\prime} \mathrm{E}$ & 400 & $\begin{array}{c}\text { Occurs, probably a } \\
\text { passage migrant }\end{array}$ \\
\hline Iran & Sahlabad Area, Khorasan & $32^{\circ} \mathrm{O} 5^{\prime} \mathrm{N} 59^{\circ} 55^{\prime} \mathrm{E}$ & 300 & $\begin{array}{l}\text { Occurs, probably as a } \\
\text { breeding bird and } \\
\text { passage migrant }\end{array}$ \\
\hline Iran & Mooteh, Isfahan & $33^{\circ} 40^{\prime} \mathrm{N} 50^{\circ} 50^{\prime} \mathrm{E}$ & 2,000 & $\begin{array}{l}\text { Regular on migration } \\
\text { (max. } 9 \text { birds) and } \\
\text { probably bred in the } \\
1970 \text { s }\end{array}$ \\
\hline Iran & $\begin{array}{l}\text { Dez River Marshes and } \\
\text { Plains, Khuzestan }\end{array}$ & $31^{\circ} 50^{\prime} \mathrm{N} 4^{\circ} 8^{\circ} 8^{\prime} \mathrm{E}$ & 200 & Winter visitor \\
\hline Iran & Kalmand, Yazd & $31^{\circ} 30^{\prime} \mathrm{N} 54^{\circ} 40^{\prime} \mathrm{E}$ & 2,419 & $\begin{array}{l}\text { An important breeding } \\
\text { area }\end{array}$ \\
\hline Iran & Kaftar Lake, Fars & $30^{\circ} 34^{\prime} \mathrm{N} 52^{\circ} 47^{\prime} \mathrm{E}$ & 47 & Five birds in August 1972 \\
\hline Iran & $\begin{array}{l}\text { Dasht-e-Arjan and Lake } \\
\text { Parishan, Fars }\end{array}$ & $29^{\circ} 34^{\prime} \mathrm{N} 51^{\circ} 53^{\prime} \mathrm{E}$ & 528 & $\begin{array}{l}\text { Occasional non-breeding } \\
\text { visitor, recorded June, } \\
\text { July and December } \\
\text { (max. } 2 \text { birds) }\end{array}$ \\
\hline Iran & $\begin{array}{l}\text { Lake Bakhtegan, lake } \\
\text { Tashk and Kamjan } \\
\text { Marshes, Fars }\end{array}$ & $29^{\circ} 40^{\prime} \mathrm{N} 53^{\circ} 30^{\prime} \mathrm{E}$ & 3,380 & $\begin{array}{l}\text { Regular non-breeding } \\
\text { visitor in late summer } \\
\text { (max. } 30 \text { in July 1974) }\end{array}$ \\
\hline Iran & Hormod, Fars & $27^{\circ} 40^{\prime} \mathrm{N} 54^{\circ} 50^{\prime} \mathrm{E}$ & 1,513 & $\begin{array}{l}\text { Regular winter visitor } \\
\text { (max. } 28 \text { in January } \\
\text { 1975) }\end{array}$ \\
\hline Iran & Bahram-e-Gour, Fars & $29^{\circ} \mathrm{Oo}{ }^{\prime} \mathrm{N} 55^{\circ} \mathrm{Oo}{ }^{\prime} \mathrm{E}$ & 3,850 & $\begin{array}{l}\text { Present all year and } \\
\text { presumably breeds. Up } \\
\text { to } 24 \text { birds recorded in } \\
\text { summer and up to } 30 \\
\text { birds in winter }\end{array}$ \\
\hline Iran & Khabr-va-Rouchoon & $28^{\circ} 51^{\prime} \mathrm{N} 56^{\circ} 28^{\prime} \mathrm{E}$ & 1,737 & $\begin{array}{l}\text { Reported to occur on } \\
\text { passage and in winter }\end{array}$ \\
\hline Iran & $\begin{array}{l}\text { Mehrouyeh, Bandar } \\
\text { Abbas }\end{array}$ & $28^{\circ} 06^{\prime} \mathrm{N} 57^{\circ} 25^{\prime} \mathrm{E}$ & 75 & $\begin{array}{l}\text { Regular winter visitor ( } 18 \\
\text { birds in 1974) }\end{array}$ \\
\hline Iran & $\begin{array}{l}\text { Kuh-i Bazman, Sistan/ } \\
\text { Baluchistan }\end{array}$ & $28^{\circ} 05^{\prime} \mathrm{N} 60^{\circ} 00^{\prime} \mathrm{E}$ & 3,247 & $\begin{array}{l}\text { Reported to occur on the } \\
\text { lower slopes in winter }\end{array}$ \\
\hline Iran & $\begin{array}{l}\text { Hamoun-i Gabi, Sistan/ } \\
\text { Baluchistan }\end{array}$ & $28^{\circ} \mathrm{O} 7^{\prime} \mathrm{N} 60^{\circ} 50^{\prime} \mathrm{E}$ & 600 & $\begin{array}{l}\text { Probably breeds (display } \\
\text { observed in 1972), and } \\
\text { winters }\end{array}$ \\
\hline
\end{tabular}


Table I. cont.

\begin{tabular}{|c|c|c|c|c|}
\hline Country & Site name & Location & $\begin{array}{l}\text { Area } \\
\left(\mathrm{km}^{2}\right)\end{array}$ & Notes \\
\hline Iran & $\begin{array}{l}\text { Kuh-i Taftan, Sistan/ } \\
\text { Baluchistan }\end{array}$ & $28^{\circ} 36^{\prime} \mathrm{N} 61^{\circ} \circ 8^{\prime} \mathrm{E}$ & 1,800 & $\begin{array}{l}\text { Recorded in suitable } \\
\text { habitat in April and so } \\
\text { may breed }\end{array}$ \\
\hline Iran & $\begin{array}{l}\text { Hamoun-i Sabari and } \\
\text { Hamoun-i Hirmand, } \\
\text { Sistan/Baluchistan }\end{array}$ & $31^{\circ} 10^{\prime} \mathrm{N} 61^{\circ} 10^{\prime} \mathrm{E}$ & 2,500 & $\begin{array}{l}\text { Regular winter visitor } \\
\text { (max. } 6 \text { birds) }\end{array}$ \\
\hline Iran & $\begin{array}{l}\text { South end of the } \\
\text { Hamoun-i Puzak, } \\
\text { Sistan/Baluchistan }\end{array}$ & $31^{\circ} 20^{\prime} \mathrm{N} 61^{\circ} 45^{\prime} \mathrm{E}$ & 149 & $\begin{array}{l}\text { One recorded in October } \\
\quad 1975\end{array}$ \\
\hline Iran & $\begin{array}{l}\text { Monde River Delta, } \\
\text { Persian Gulf Coast }\end{array}$ & $28^{\circ} 10^{\prime} \mathrm{N} 51^{\circ} 18^{\prime} \mathrm{E}$ & 467 & $\begin{array}{l}\text { Regular winter visitor } \\
\text { (max. 40 birds in } \\
\text { January 1975) }\end{array}$ \\
\hline Iran & $\begin{array}{l}\text { Rud-i-Jagin and } \\
\text { Rud-i-Gabrik Deltas, } \\
\text { Sistan/Baluchistan }\end{array}$ & $25^{\circ} 35^{\prime} \mathrm{N} 58^{\circ} 20^{\prime} \mathrm{E}$ & 140 & $\begin{array}{l}\text { Possibly a winter visitor; } \\
\text { one bird recorded in } \\
\text { March } 1971\end{array}$ \\
\hline Iran & $\begin{array}{l}\text { Lower Sarbaz River and } \\
\text { Khor Govater, Sistan/ } \\
\text { Baluchistan }\end{array}$ & $25^{\circ} 25^{\prime} \mathrm{N} 61^{\circ} 15^{\prime} \mathrm{E}$ & 3,824 & $\begin{array}{l}\text { Regular winter visitor to } \\
\text { plains of Dasht-i-Ari } \\
\text { (max. I1 birds in March } \\
\text { 1971) }\end{array}$ \\
\hline Iraq & $\begin{array}{l}\text { Mahzam and Lake } \\
\text { Tharthar, Salah Al Din }\end{array}$ & $34^{\circ} 20^{\prime} \mathrm{N} 43^{\circ} 22^{\prime} \mathrm{E}$ & 4,550 & $\begin{array}{l}\text { Resident or summer } \\
\text { visitor ( } 2 \text { "pairs") }\end{array}$ \\
\hline Iraq & $\begin{array}{l}\text { Samara Dam, Salah Al } \\
\text { Din }\end{array}$ & $34^{\circ} 15^{\prime} \mathrm{N} 43^{\circ} 50^{\prime} \mathrm{E}$ & 200 & Has bred in the area \\
\hline Iraq & $\begin{array}{l}\text { Abu Dalaf and Shari/ } \\
\text { Lake, Salah Al Din }\end{array}$ & $34^{\circ} 15^{\prime} \mathrm{N}_{44^{\circ}}^{\circ} 0^{\prime} \mathrm{E}$ & 1,270 & $\begin{array}{l}\text { Has occurred, possibly } \\
\text { breeds }\end{array}$ \\
\hline Iraq & Attariya Plains, Baghdad & $33^{\circ} 25^{\prime} \mathrm{N} 44^{\circ} 55^{\prime} \mathrm{E}$ & 500 & $\begin{array}{l}\text { Seen in April; possibly } \\
\text { breeds }\end{array}$ \\
\hline Israel & Western Negev & $31^{\circ} 10^{\prime} \mathrm{N} 34^{\circ} 40^{\prime} \mathrm{E}$ & 900 & $\begin{array}{l}\text { The main breeding area in } \\
\text { Israel with up to } 50 \\
\text { "pairs", chiefly in the } \\
\text { Ze'elim and Agur } \\
\text { Sands area }\end{array}$ \\
\hline Israel & $\begin{array}{l}\text { Cliffs of Zin and the } \\
\text { Negev Highlands }\end{array}$ & $30^{\circ} 50^{\prime} \mathrm{N} 34^{\circ} 4^{8^{\prime} \mathrm{E}}$ & 250 & $\begin{array}{l}\text { Up to } 3 \text { "pairs" breed and } \\
\text { a maximum of ro birds } \\
\text { winter }\end{array}$ \\
\hline Jordan & Burqu, Mafraq & $32^{\circ} 42^{\prime} \mathrm{N} 37^{\circ} 57^{\prime} \mathrm{E}$ & 950 & $\begin{array}{l}\text { May breed in the } \\
\text { inaccessible harrat, and } \\
\text { almost certainly winters }\end{array}$ \\
\hline Jordan & Azraq Oasis, Zarqa & $31^{\circ} 49^{\prime} \mathrm{N} 36^{\circ} 4^{8^{\prime} \mathrm{E}}$ & 120 & $\begin{array}{l}\text { Formerly a resident in less } \\
\text { disturbed areas of } \\
\text { desert just east of Qa al } \\
\text { Azraq; may still occur } \\
\text { as an occasional winter } \\
\text { visitor, although there } \\
\text { are no recent known } \\
\text { sightings }\end{array}$ \\
\hline Jordan & Shaumari Area, Zarqa & $31^{\circ} 48^{\prime} \mathrm{N} 36^{\circ} 49^{\prime} \mathrm{E}$ & 342 & $\begin{array}{l}\text { In the 1970s it was } \\
\text { thought to breed (2+ } \\
\text { "pairs") and winter } \\
\text { visitor (max. 1o birds). } \\
\text { No more recent records. }\end{array}$ \\
\hline Jordan & $\begin{array}{l}\text { Hisban-Madaba Plains, } \\
\text { Amman }\end{array}$ & $31^{\circ} 45^{\prime} \mathrm{N} 35^{\circ} 47^{\prime} \mathrm{E}$ & 45 & $\begin{array}{l}\text { Potentially a rare winter } \\
\text { visitor to uncultivated } \\
\text { areas }\end{array}$ \\
\hline
\end{tabular}


Table 1. cont.

\begin{tabular}{|c|c|c|c|c|}
\hline Country & Site name & Location & $\begin{array}{l}\text { Area } \\
\left(\mathrm{km}^{2}\right)\end{array}$ & Notes \\
\hline Jordan & Wadi Araba, Ma'an & $30^{\circ} 05^{\prime} \mathrm{N} 35^{\circ} 11^{\prime} \mathrm{E}$ & 1,500 & $\begin{array}{l}\text { Probably a rare resident } \\
\text { or winter visitor ( } 4 \text { seen } \\
\text { to the north near Fifa in } \\
\text { winter 1982) }\end{array}$ \\
\hline Kuwait & Al-Batin 'Park', Al-Jahra & $29^{\circ} 15^{\prime} \mathrm{N}_{4} 6^{\circ} 51^{\prime} \mathrm{E}$ & 575 & Formerly bred \\
\hline Oman & Masirah, Ash Sharqiyah & $20^{\circ} 25^{\prime} \mathrm{N} 58^{\circ} 47^{\prime} \mathrm{E}$ & 1,095 & $\begin{array}{l}\text { According to local people, } \\
\text { regular passage migrant } \\
\text { in autumn with up to } 4 \\
\text { per day in October }\end{array}$ \\
\hline Oman & $\begin{array}{l}\text { Jiddat al Harasis, Al } \\
\text { Wustta }\end{array}$ & $19^{\circ} 48^{\prime} \mathrm{N} 56^{\circ} 49^{\prime} \mathrm{E}$ & 27,500 & $\begin{array}{l}\text { Frequently recorded } \\
\text { resident and winter } \\
\text { visitor; thought to be } \\
\text { the most important } \\
\text { breeding site in Oman }\end{array}$ \\
\hline Oman & Khawr Dhirif, Al Wustta & $18^{\circ} 56^{\prime} \mathrm{N} 57^{\circ} 21^{\prime} \mathrm{E}$ & 1 & $\begin{array}{l}\text { Rare winter visitor in the } \\
\text { surrounding desert ( } 2 \\
\text { recorded in October) }\end{array}$ \\
\hline Saudi Arabia & Harrat al-Harrah, al Jawf & $31^{\circ} \mathrm{oo} \mathrm{N}_{3} 8^{\circ} 5 \mathrm{o}^{\prime} \mathrm{E}$ & 13,775 & $\begin{array}{l}\text { Breeds in low numbers } \\
\text { (max. } 50 \text { breeding } \\
\text { females), and wintering } \\
\text { area (max. } 570 \text { in } \\
\text { November) }\end{array}$ \\
\hline Saudi Arabia & $\begin{array}{l}\text { Al-Hasa Lagoons, Eastern } \\
\text { Province }\end{array}$ & $25^{\circ} 30^{\prime} \mathrm{N} 50^{\circ} \mathrm{oo} \mathrm{E}$ & 75 & $\begin{array}{l}\text { According to local people, } \\
\text { regular in small } \\
\text { numbers on passage } \\
\text { and in winter }\end{array}$ \\
\hline Saudi Arabia & $\begin{array}{l}\text { Hawtat Bani Tamim, } \\
\text { Ar-Riyadh }\end{array}$ & $23^{\circ} 30^{\prime} \mathrm{N} 46^{\circ} 30^{\prime} \mathrm{E}$ & 2,369 & Rare, non-breeding visitor \\
\hline Saudi Arabia & Mahazet as-Sayd, Makkah & $22^{\circ} 15^{\prime} \mathrm{N} 41^{\circ} 5^{\prime} \mathrm{E}$ & 2,190 & $\begin{array}{l}\text { A few birds occur in } \\
\text { winter; site of a } \\
\text { reintroduction scheme }\end{array}$ \\
\hline Saudi Arabia & $\begin{array}{l}\text { Al-Habrow al-Arabi, } \\
\text { Makkah, Asir }\end{array}$ & $18^{\circ} 40^{\prime} \mathrm{N} 41^{\circ} 30^{\prime} \mathrm{E}$ & 2,500 & $\begin{array}{l}\text { Regular winter visitor in } \\
\text { small numbers }\end{array}$ \\
\hline Syria & Tual al-'Abba, Al-Raqqah & $36^{\circ} 25^{\prime} \mathrm{N} 39^{\circ} 20^{\prime} \mathrm{E}$ & 300 & $\begin{array}{l}\text { Said to have been a } \\
\text { former visitor to the } \\
\text { region, driven away by } \\
\text { excessive hunting }\end{array}$ \\
\hline Syria & $\begin{array}{l}\text { Jabal Abdul Aziz, } \\
\text { Al-Hasakah }\end{array}$ & $35^{\circ} 25^{\prime} \mathrm{N} 40^{\circ} 22^{\prime} \mathrm{E}$ & 450 & Resident \\
\hline Syria & $\begin{array}{l}\text { Buhayrat al-Khatuniyah, } \\
\text { Al-Hasakah }\end{array}$ &  & 800 & Resident \\
\hline Syria & Sabkhat al-Jabbul, Halab & $36^{\circ} \mathrm{o}^{\prime} \mathrm{N} 37^{\circ} 29^{\prime} \mathrm{E}$ & 15 & $\begin{array}{l}\text { Resident on surrounding } \\
\text { steppe }\end{array}$ \\
\hline Syria & $\begin{array}{l}\text { Dayr al-Zawr, Euphrates } \\
\text { Valley }\end{array}$ & $35^{\circ} 23^{\prime} \mathrm{N} 40^{\circ} 07^{\prime} \mathrm{E}$ & & $\begin{array}{l}\text { Breeds on the steppe in } \\
\text { the vicinity }\end{array}$ \\
\hline Syria & Wadi al-Azib, Hamah & $35^{\circ} 36^{\prime} \mathrm{N} 37^{\circ} 20^{\prime} \mathrm{E}$ & 242 & Resident \\
\hline Syria & $\begin{array}{l}\text { Jabal al-Bishri, Dar } \\
\text { al-Zawr }\end{array}$ & $35^{\circ} 20^{\prime} \mathrm{N} 39^{\circ} 30^{\prime} \mathrm{E}$ & 20 & Resident \\
\hline Syria & Jabal al-Bilas, Hims & $34^{\circ} 55^{\prime} \mathrm{N} 37^{\circ} 55^{\prime} \mathrm{E}$ & 400 & $\begin{array}{l}\text { Breeds, and also a winter } \\
\text { visitor }\end{array}$ \\
\hline Syria & $\begin{array}{l}\text { Tadmur and Sabkhat } \\
\text { Muh, Hims }\end{array}$ & $34^{\circ} 30^{\prime} \mathrm{N} 38^{\circ} 18^{\prime} \mathrm{E}$ & 450 & $\begin{array}{l}\text { Apparently breeds in the } \\
\text { surrounding desert }\end{array}$ \\
\hline Syria & Jabal Sis, Dimashq & $33^{\circ} 18^{\prime} \mathrm{N} 37^{\circ} 22^{\prime} \mathrm{E}$ & 400 & $\begin{array}{l}\text { Adult seen in May 1976; } \\
\text { certain to be breeding }\end{array}$ \\
\hline
\end{tabular}


Table 1. cont.

\begin{tabular}{lllcl}
\hline Country & Site name & Location & $\begin{array}{c}\text { Area } \\
\left(\mathrm{km}^{2}\right)\end{array}$ & Notes \\
\hline $\begin{array}{c}\text { United Arab } \\
\text { Emirates }\end{array}$ & $\begin{array}{c}\text { Digdaga-Hamraniyah, Ras } \\
\text { al Khaimah }\end{array}$ & $25^{\circ} 40^{\prime} \mathrm{N} \quad 55^{\circ} 55^{\prime} \mathrm{E}$ & 20 & $\begin{array}{c}\text { Formerly a regular winter } \\
\text { visitor to nearby Jiri } \\
\text { plain, but no records } \\
\text { since } 1980\end{array}$ \\
$\begin{array}{c}\text { United Arab } \\
\text { Emirates }\end{array}$ & Zabeel Ponds, Dubai & $25^{\circ} 12^{\prime} \mathrm{N} 55^{\circ} 18^{\prime} \mathrm{E}$ & $\begin{array}{c}\text { Said to be common in } \\
\text { winter in desert to }\end{array}$ \\
$\begin{array}{c}\text { United Arab } \\
\text { Emirates }\end{array}$ & Baynunah, Abu Dhabi & $23^{\circ} 57^{\prime} \mathrm{N} 52^{\circ} 39^{\prime} \mathrm{E}$ & 1,800 & $\begin{array}{c}\text { Wouth } \\
\text { Winter visitor }\end{array}$ \\
$\begin{array}{c}\text { Yemen } \\
\text { Desert west of Al-Ghayda, } 16^{\circ} 13^{\prime} \mathrm{N} 51^{\circ} 49^{\prime} \mathrm{E}\end{array}$ & 50 & $\begin{array}{c}\text { Reported to have } \\
\text { wintered in the area } \\
\text { previously, and may }\end{array}$ \\
\hline
\end{tabular}

Source: Evans (1994).

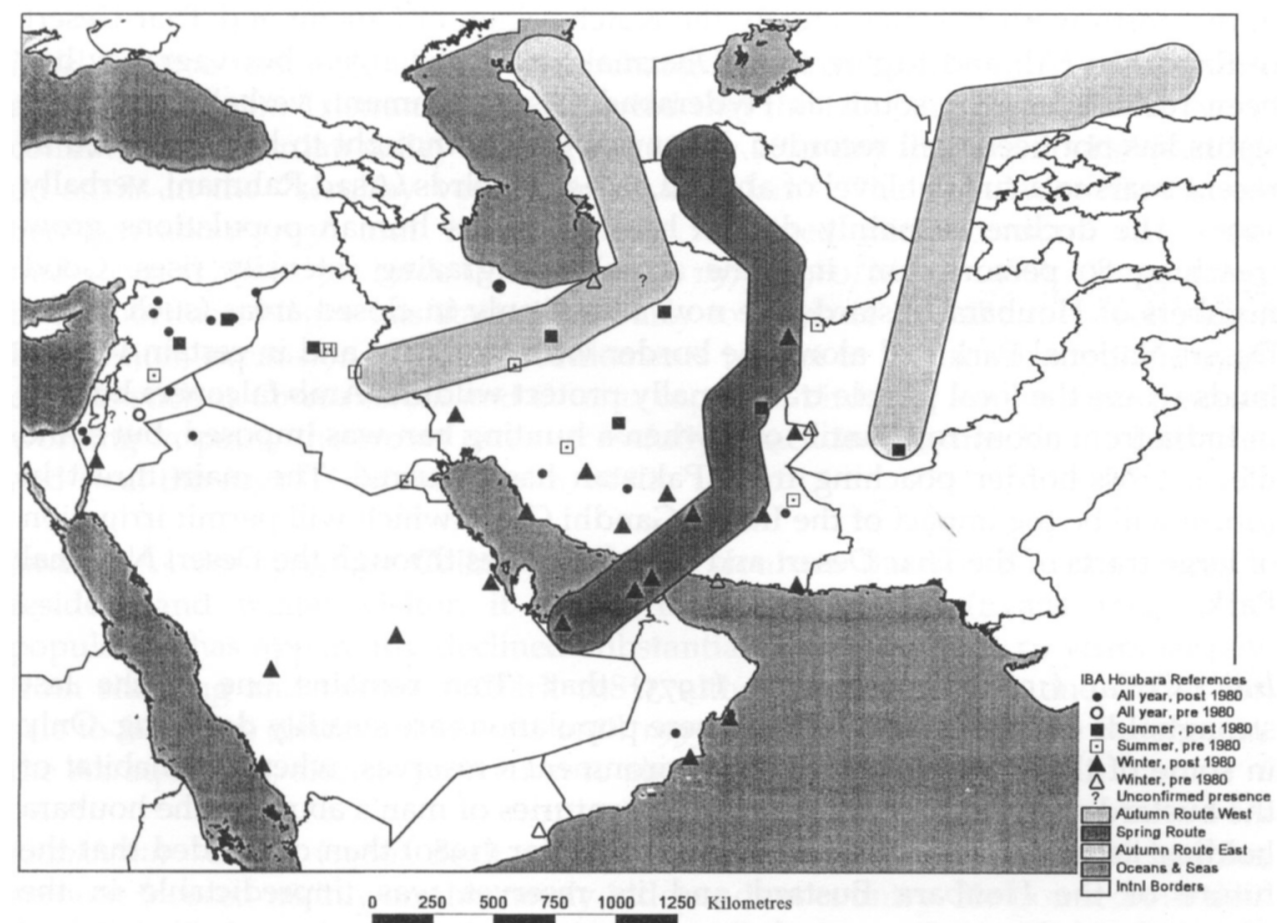

Figure 2. Broad movements of the Asian Houbara Bustard based on preliminary satellite tracking data from the National Avian Research Centre (Abu Dhabi, U.A.E.), with Important Bird Areas (IBAs) where Houbara Bustards have been recorded (see Table 1) after Evans (1994). Note the close coincidence of the migration paths and known IBA sites. 
record was from the western region of Xinjiang about $10 \mathrm{~km}$ south of the Aqal oasis (c. $42^{\circ} 10^{\prime} \mathrm{N}, 80^{\circ} 00^{\prime} \mathrm{E}$ ) by Grimmett and Taylor (1992). In surveys during 1994 by the Chinese Academy of Sciences (Gao Xing-Yi, in litt. 1995), suitable habitat totalling $14,900 \mathrm{~km}^{2}$ held an estimated 280 to 525 birds.

Egypt Collar (1980) found recent information to be very feeble. According to Meinertzhagen (1930), it was frequently observed on the plains of northern Sinai, where it was believed to be a rare resident, but the majority of birds were almost certainly winter visitors. It is still resident in the plains of the Sinaï peninsula (Saleh 1989) and a winter visitor in some numbers. In 1990, a Saudi Arabian falconer, HRH Prince Abdul Rahman al Faisal, had obtained a long-term lease (for about US\$25,000 per year) to hunt in the northern part of the Sinai peninsula, which included employing local people as wardens to prevent "poaching" (Governor of Sinai, verbally 1990); he was catching about 140 birds per year.

India Collar (1980) described the information available from India as mainly anecdotal accounts by colonial British writers about hunting wintering birds, with no real indication of status. The Houbara Bustard is a regular winter visitor in the north-western states, chiefly the Kutch region of Gujarat and Thar Desert of Rajasthan (Ali and Ripley 1969, Dharmakumarsinhji 1955), but vagrancy has been recorded as far south as Hyderabad (Asad Rahmani, verbally 1996). Its status has not been well recorded, but numbers are thought to have declined in recent years to a current level of about 2,000-5,000 birds (Asad Rahmani, verbally 1996). The decline is mainly due to habitat loss as human populations grow (reaching 80 persons $/ \mathrm{km}^{2}$ in some areas) and grazing intensity rises. Good numbers of Houbara Bustards are now found only in closed areas (such as the Desert National Park and along the border with Pakistan) and in certain Vishnoi lands where the local people traditionally protect wildlife. Arab falconers hunted in India from about 1974 until 1980, when a hunting ban was imposed, but some illegal cross-border poaching from Pakistan has occurred. The main threat in future will be the impact of the Indira Gandhi Canal which will permit irrigation of large tracts of the Thar Desert and actually passes through the Desert National Park.

Iran Collar (1980) quotes Scott (1975) that "Iran remains one of the last strongholds of the species, yet even here populations are steadily declining. Only in some of the Department of the Environment's reserves, where the habitat of the bustards is slowly regenerating after centuries of man's abuse, is the houbara holding its own and perhaps increasing"; Collar (1980) then concluded that the future of the Houbara Bustard and its reserves was unpredictable in the aftermath of the 1978-79 revolution. Recent information suggests that it is a resident, passage migrant (from west Kazakhstan) and winter visitor across a wide tract of the country, including 24 IBA sites (Razdan and Mansoori 1989; Evans 1994). It appears that the resident population has suffered a severe decline over the past 30 years to become generally scarce: the best area in the country is in the protected region of Kalmand $\left(1,500 \mathrm{~km}^{2}\right)$ where some $200-500$ birds breed. The Department of the Environment is planning to establish a research centre in this region (Jamshed Mansoori, verbally 1996). The number of birds visiting the 
country in winter has not been assessed. The main threats are habitat loss and deterioration, overgrazing and hunting (although Arab falconry parties have visited much less often following the revolution) but some populations fall within the still existing protected areas.

Iraq Collar (1980) concluded that the status of the Houbara Bustard was unclear. It was apparently locally common in the north and west of the country, perhaps migratory in the south and centre, during the early part of this century (Meinertzhagen 1924, Moore and Boswell 1956, Ticehurst et al. 1922) Later, however, Marchant (1961) suggested that it was no longer common and had decreased rapidly since the 1920s. Recent sources suggest that the Houbara Bustard is a scarce resident, occurring in four IBA sites (Evans 1994) and passage migrant and winter visitor. A female caught in the Mangyshlak Peninsula (western Kazakhstan) was tracked by satellite to a locality in eastern Iraq, close to the IBA site in the Attariya Plains (F. Launay, verbally 1995). There continue to be regular hunts along the Iraqi borderlands by falconers from Kuwait and Saudi Arabia, with one report received by the author of some 2,000 birds killed during winter 1994 .

Israel Scarce resident but some interchange of birds likely with Syria, Jordan and Egypt (Sinaii). Tristram (1868) reported that the Houbara Bustard was "very common in the Jordan valley", being seen "day after day in small flocks" feeding on snails in the "scrubby vegetation of the plain". The population fluctuated between about 1930 and 1975, but has remained fairly constant since the mid-1970s at about 300 birds (Mendelssohn 1975, 1980, Mann 1986). Overgrazing in the northern Negev has started seriously to degrade its habitat (Lavee 1983). In addition, expansion of human settlements has let to increased populations of corvids such as Corvus corone and C. corax (up to 34 birds per $\mathrm{km}^{2}$ in some places); working in pairs, the crows can successfully attack incubating female bustards and steal the eggs (H. Mendelssohn, in litt. 1992).

Jordan As described by Collar (1980), the Houbara Bustard remains a scarce resident and winter visitor; it occurs in five IBA sites (Evans 1994). The population has apparently declined substantially over the past 50 years largely from shooting and falconry (Clark 1983), although trapping by one trader for sale to falconers continued at least until 1993. The Shaumari Wildlife Reserve, in the Azraq region, affords protection to about 30 birds (Clark 1983).

Kazakhstan Collar (1980) dealt with the former U.S.S.R. as a whole, but his account focused mainly on this newly independent country. He pointed out that declines in the population had already been reported long before hunting started in Pakistan. Thus, Dolgushin (1962) wrote: "The number of bustards at present is decreasing everywhere. Not only has excessive persecution by man had an unfavourable effect on their numbers, but also the intensive ploughing-up of steppe. In several places, particularly near large industrial centres, the numbers of houbaras has noticeably decreased owing to uncontrolled shooting of birds from motor vehicles. The periodical fluctuation of houbara numbers has not been studied, but undoubtedly their occurrence is the result of loss and depletion 
through starvation in their winter quarters in severe winters. Thus in the cold winter of 1919 near Ashkhabad [Turkmenistan] a mass mortality among the wintering houbaras was recorded; exhausted birds even flew into suburban gardens'. Rozanov (1977) added that extensive development of (and within) desert areas had led to overgrazing and habitat degradation by domestic animals, coupled with road construction, industrial growth, settlements, drilling and mining, canal-building and irrigation schemes, and the consumption of surface and groundwater for agricultural, industrial and domestic needs exerted an intolerable pressure on the breeding grounds of the Houbara Bustard. Subsequently, the Soviet Red Data Book (1978) listed the species as very rare, and E. E. Syroietchovsky (verbally to N. J. Collar, 1978) estimated the total U.S.S.R. population at only a few thousand birds.

In stark contrast, it is now known that the Houbara Bustard is a locally common breeding summer visitor. Investigations over a 10-year period in the Kyzyl Kum by Gubin (1986), latterly using ground and aerial surveys, resulted in an estimated population of around 2,50o birds for the region, and this led to an estimate of 30,000-40,000 birds for the country as a whole (B. Gubin, Kazakhstan National Report to CMS, 1994). The large discrepancy between the contemporary situation and that previously reported could mean (i) the decline was highly localized and much less severe than thought; (ii) there was a significant decline but the former population must have been very much higher than was ever considered; (iii) there was a decline but some recovery took place as economic decline set in. In any case, Kazakhstan is by far the most important range state for the Houbara Bustard. In 1995, a formal Memorandum of Research Cooperation was signed between the National Academy of Sciences and the National Avian Research Center, Abu Dhabi. Under this Memorandum, field research was carried out in about $1,400 \mathrm{~km}^{2}$ of the Taukum sands, south of Lake Balkash. The researchers registered 32-35 displaying male Houbara Bustards, located seven nests and found 12 females with chicks, trapped seven males and fitted them with satellite transmitters, and collected 16 chicks for a joint captive breeding scheme. The overall population in the area was estimated at 120 birds (F. Launay and B. Gubin, in litt. 1996). The satellite tracking data (Figure 2) suggest that birds breeding in the west of the country tend to move south-west to Iran and Iraq, while those breeding in the east migrate first westwards, skirting the Tian Shan mountains, then continue southwards to Uzbekistan, Turkmenistan, Afghanistan, Pakistan, India and the Arabian peninsula. One migratory group in September 1995 comprised 49 birds. One falconer from Saudi Arabia was permitted to hunt in 1994, but caught only a few birds before a public outcry led to the licence being revoked ("Noah" 1994).

Kuwait Rare passage migrant and winter visitor. It formerly bred around Al-Jahra, an IBA site (Evans 1994). A report of about 20 birds hunted in the north of the country in November 1995 is the first confirmed record in recent times (Samira Omar, verbally 1996). Threats include drought, disturbance, overgrazing and general environmental deterioration in the aftermath of the Gulf War.

Mongolia Uncommon breeding visitor (July to September) ranging from Ulaangom in the north-west, south-east to south of Dalandzadgad; there have 
been sightings totalling about 50 birds between 1975 and 1980 , and there seems to have been a decline in recent years (D. Batdelger, in litt. 1995).

Oman Collar (1980) simply referred to Stanford (1973) who described the Houbara Bustard as a widespread winter visitor but apparently also resident, breeding south of Ibri during the period from 1954 to 1968. It is now considered to be an uncommon breeding resident numbering several hundred to a few thousand birds, occurring in the Jiddat al Harasis, Rub Al Khali and Ja'alan, and also a passage migrant and winter visitor from August to March in small, irregular numbers (Eriksen 1996, Gallagher and Woodcock 1980, Oman Bird Records Committee 1994). The resident population appears to be stable, but the migratory visitors may be declining due to hunting in the countries of origin (Oman National Report to CMS, 1995). Until 1995, Baraimi market in the Oman sector of $\mathrm{Al}$ Ain was reported to be a major entrepôt for selling illegally imported Houbara Bustards to falconers from U.A.E. although the authorities have recently taken steps to prevent further trading. Hunting of Houbara Bustards is not permitted (Oman National Report to CMS, 1995), and measures have been taken to prevent poaching by parties from neighbouring countries. Local disturbance and habitat degradation from activities such as oil exploration could be having a small impact on the resident population.

Pakistan Collar (1980) noted that the Houbara Bustard might breed in very small numbers, and that it was a winter visitor from the former U.S.S.R. He pointed out the large discrepancy between the old accounts of the bird's status and the numbers that were later killed by visiting Arab hunters. Reports of declines and warnings of extinction were published, but no hard evidence emerged to indicate that falconry alone was having a major impact on Houbara Bustard numbers, given that it was already extensively hunted by local people. It can be considered a very rare resident, and locally common and widespread passage migrant and winter visitor from August to March. The breeding range is confined to Baluchistan comprising some $4,500 \mathrm{~km}^{2}$ in northern Chaigai, $3,750 \mathrm{~km}^{2}$ in Rakshan and $1,250 \mathrm{~km}^{2}$ in Kharan, but breeding records are generally sporadic (Mian et al. 1988). Probably the most important breeding area is in the Nag region of Kharan, where there were about 60 birds in 1995 (A. Virk, in litt. 1995) but they may have declined since to even lower numbers according to the poor results of a survey in April 1997 (B. Gubin and E. Mukhina in litt. 1997). Eggs and chicks were collected from here for a breeding scheme at the National Wildlife Research Centre at Taif in Saudi Arabia in 1987 and 1988 (Paillat et al. 1988) and some collecting of eggs by local people still continues in the hope of selling them. The numbers of birds arriving in the country for the winter period (October to March) is the subject of much conjecture and no doubt highly variable depending on weather conditions further north and whether birds divert to Iran or India, or continue south to Arabia for the winter (Pakistan National Report to CMS, 1995). For example, Goriup (1982) estimated that the wintering population in Pakistan as a whole might be somewhere between 16,000 and 20,000 birds; Mian et al. (1988). claimed that the wintering population in Baluchistan alone dropped from 22,000 birds in $1983 / 1984$ to 7,500 birds in $1987 /$ 1988 almost entirely as a result of hunting pressure; but in November/December 
1994, surveys at three sites in Baluchistan suggested a wintering population of over 19,000 birds, with a further 3,700 in Punjab and 750 in Sindh (Chaudhry 1996). This variation can in large part be explained by the availability of vast areas for wintering Houbara Bustards, only a tiny fraction of which can be surveyed by the investigators involved; when small changes in apparent density (bearing in mind that the birds tend to clump in small flocks rather then spread out evenly) are multiplied by the area concerned (over $166,000 \mathrm{~km}^{2}$ according to Chaudhry 1996), a large margin of error is inevitable. In the absence of consistent, coordinated, wide-ranging surveys conducted over several years, the wintering population level and its dynamics will remain a mystery.

Since the mid-1960s, Pakistan has hosted falconers from the Gulf countries who come to hunt the Houbara Bustard under special licences issued by the Ministry of Foreign Affairs (it is protected under national law) and who have been allocated personal hunting areas (Mirza 1971; Pakistan National Report to CMS, 1995). These include HH Sheikh Zayed bin Sultan al-Nahyan (Ruler of Abu Dhabi and President of U.A.E.), HH Sheikh Khalifa bin Hamad al-Thani from Qatar and HRH Prince Naif bin Abdul Aziz from Saudi Arabia (Chaudhry 1996). Until the mid-1980s, the hunting parties between them were estimated to kill between 3,000 and 7,000 birds each year (Kroll 1977, Goriup 1982, Mian 1986, Pakistan National Report to CMS, 1995) but that after 1987 there was a severe reduction in the bag to a few hundred birds (Pakistan National Report to CMS, 1995), although this is not substantiated by falconers themselves in discussion with the author. In addition, there is a considerable amount of poaching by local hunters and extensive trapping and export to provide falconers with Houbara Bustards on which to train their falcons (Pakistan National Report to CMS 1995): this harvest is thought to amount to between 4,000 and 7,000 more birds (Brig. M. Ahmed, verbally 1996). This level of taking has led many conservationists to question the sustainability of the current hunting regime (including trade for training) and various studies have been carried out (e.g. Goriup 1982, Roberts and Savage 1971, Mian et al. 1988, Mirza 1971, and see Bustard Studies vol. 3) supported by the Pakistan government and the World Wide Fund for Nature (WWF). This work expanded in November 1995 when WWF-Pakistan signed a formal Memorandum of Research Cooperation with the Institute of Zoology at Tashkent in Uzbekistan.

Qatar Rare passage migrant and winter visitor.

Russia Probably an occasional summer visitor south of the Volga, around the north-west coast and hinterland of the Caspian Sea, and perhaps occurring in the Tuva region, contiguous with the Mongolian population. It was a regular summer visitor to these areas in the early part of this century (Ponomareva 1979), recorded as far north as Saratov and as far west as the Krasnodar region near the Black Sea in winter 1962 and May 1966 (Ochaporskiy 1986).

Saudi Arabia Collar (1980) quoted D. Ferguson (in litt. 1977) as writing: ". . . the houbara is now probably and at least virtually extinct as a breeding species although some may still occur on the Summan plateau in the al-Habl area ... in general, wintering birds are still so few that no-one troubles to mount hunts for 
them, electing instead to travel abroad to do so". However, Collar (1980) was informed by J. E. Burchard (verbally, 1979) that the King of Saudi Arabia still successfully took large numbers of Houbara Bustards in the winter hunting season and that there was reliable information that birds still bred in some areas. The bird's status remains as a rare breeding resident (probably less than 500 birds) and regular winter visitor from October to March in variable but unknown numbers and recorded in five IBA sites (Evans 1994, Seddon et al. 1995); however, several hundred birds are reported to be killed by hunters each winter in the plains bordering Iraq and Kuwait between Hafr al Batin and Turaif. The only known breeding sites at present are in Harrat al Harrah (Goriup et al. 1992), a protected area declared in 1987 lying close to the Jordan border, the Summan plateau and along the eastern fringes of the Dahna sands and Rub 'al-Khali (Jennings 1995). Wintering birds seem to occur sporadically almost throughout the country in suitable habitats, including inside the Rub 'al-Khali and crossing the Asir escarpment to reach the Tihama plain south of Jeddah (Felemban and al Salamah 1992). A captive breeding and release programme was initiated at the National Wildlife Research Centre at Taif (a branch of the National Commission for Wildlife Conservation and Development) in 1986, and between 1991 and 1995, 135 birds were released which resulted in the successful establishment of 53 captive-bred birds and breeding by two females in the Mahazet Asaid protected area near Taif. Another 40 to 50 subadult birds were released during 1996 (Seddon et al. 1995, Seddon and Maloney 1996, Saudi Arabia National Report to CMS, 1996). The management priorities for the Houbara Bustard in Saudi Arabia have been set out in a species conservation strategy (Seddon 1995). These include plans to expand the release programme to other suitable protected areas, and the further development of an agreement for the Houbara Bustard under the Bonn Convention (HRH Prince Saud al Faisal 1990, Saudi Arabia National Report to CMS, 1996).

Syria Collar (1980) provides a translation of Kumerloeve (1968) as follows: "Still quite numerous as a nesting species, and distributed throughout the Syrian desert and semi-desert; but generally in decline owing to massive persecution". It can be considered to be a scarce resident and winter visitor. It occurs (or used to occur) in 10 IBA sites (Evans 1994) but all recent accounts suggest that the population has declined severely during the last 50 years from habitat degradation, disturbance and hunting by local people (Macfarlane 1978, Baumgart et al., 1995, Roshier 1995). One bird marked in Harrat al Harrah, Saudi Arabia in November 1990 (Goriup et al. 1992) was killed by a hunter in Syria in 1993 (H. A. Abuzinada in litt. 1993).

Tadjikistan Rare summer visitor, known only from the lower reaches of the Kalirnagan River and the southern side of the Syr Darya River (Oleg Mitropolsky, verbally 1996).

Turkey The presence of Houbara Bustards in Armenia and possibly Azerbaijan implies that they perhaps could still occur in the extreme east of Turkey where it is thought to be extinct (Goriup and Parr 1985): however, there is certainly 
suitable habitat between Çalpak and Küllük, and around Mount Ararat (pers. obs. 1981).

Turkmenistan Summer visitor and locally common passage migrant; possibly occurs throughout the year. The status of the Houbara Bustard is not documented, but the preliminary results of satellite tracking work by the National Avian Research Center confirms that the geographic situation of the country means that the bulk of the Central Asian population must pass through it during spring and autumn. There have already been attempts by falconers from U.A.E. to hunt in the country but they were refused permits and fined $\$ 40,000$ for poaching (Zatoka 1995).

United Arab Emirates Collar (1980) considered that small numbers of Houbara Bustards passed through in winter, while the breeding population, if it existed, was very feeble. According to C. D. W. Savage (in litt. to N. J. Collar, 1979), numbers may have declined partly as a result of locust control programmes, but the evidence for this was scant and imprecise. It is still a scarce passage migrant and winter visitor across much of the country with $300-600$ birds occurring between late September and mid-March (M. al Bowardi, verbally 1995). While a few birds over-summer, there is no confirmed evidence of breeding (U.A.E. National Report to CMS, 1994, Osborne 1996) probably because the mean temperature during the breeding season is too high. It appears that the numbers available for hunting have greatly declined since the 1960s, when it was possible to catch many birds even close to Abu Dhabi city ( $R$. Upton, verbally 1995), probably as a result of increased human activity in the desert regions (particularly oil prospecting and joy-riding), gradual loss of vegetation and expansion of grazing from settlements (U.A.E. National Report to CMS, 1994). Hunting of Houbara Bustards is allowed only in designated areas, either privately owned or in a so-called public hunting area of about $11,000 \mathrm{~km}^{2}$ situated to the south of Dubai (U.A.E. National Report to CMS, 1994). A pilot survey of 15 falconers from the Emirates suggested that 307 Houbara Bustards had been seen by them in 1994/1995, of which they said $70 \%$ had been caught (T. Bailey, in litt. 1995). In 1989, the National Avian Research Center was established in Abu Dhabi to carry out research on and management of Houbara Bustards, falcons and other birds in the country as well as in other appropriate range states (Bailey and Hornby 1994). In spring 1995, a Houbara Bustard fitted with a satellite transmitter was tracked moving from western U.A.E., through eastern Iran to a location in north-west Turkmenistan (Figure 2), the first successful long-range tracking of this species.

Uzbekistan Locally common summer visitor (from late March to mid-November) and passage migrant (occasionally over-wintering), mainly in the western part of the country (Karakalpakstan) as far east as Bukhara (Ponomareva 1985, Mukhina 1989, Mitropolsky et al. 1996); in the Uch Kuduk area, population densities vary from 0.5 to 0.6 birds per $\mathrm{km}^{2}$ (Launay and Loughland 1995). According to Mitropolsky et al. (1996), the overall population in Uzbekistan comprises some 2,200-2,700 nesting females (assuming an even sex ratio and one-third of the population subadult, this would mean an overall 
population of about 6,000-9,000 birds). Nevertheless, in the early part of this century (before the mid-1950s), the Houbara Bustard was more widespread and abundant than now, breeding throughout the Kyzyl Kum desert wherever suitable habitat occurred up to about $600 \mathrm{~m}$ altitude. Early census work in the Kyzyl Kum region by Alekseev (1980) suggested that the population there was relatively stable between 1956 and 1967, but then rapidly declined by between $66 \%$ and $75 \%$ by 1979 . However, the data obtained suffer from changes in method over the period so while a decline was clearly evident, its true extent is uncertain. The main reason for its recent decline in range has been habitat loss to irrigated agriculture (including aerial application of pesticides), livestock production and other forms of rural development (Mitropolsky et al. 1996). One protected area covering 30,000 ha in Karabchul was established to protect the Houbara Bustard, but there is no recent information about the status of this area (Mitropolsky et al. 1996). The Houbara Bustard is a protected species and hunting it is illegal, but there is some local poaching and egg-taking by herdsmen. Falconers from Arabia are known to have paid visits in autumn 1993 (taking at least 40 birds) and again in autumn 1994 (Mitropolsky et al. 1996). An application from Bahraini falconers to hunt in winter 1996 was refused (Mukhina in litt. 1997).

Yemen Uncommon winter visitor with a small breeding population reported to be present (Jennings 1995, Osborne 1996). Houbara Bustards are caught by local people for sale and for food, and there are visiting parties of hunters from neighbouring countries (O. al-Saghier, verbally 1996). There are no protection measures for the Houbara Bustard at present.

\section{Vagrancy}

Houbara Bustards have been recorded sporadically, and not less than to years ago, in Belgium, Cyprus, Slovak Republic, Denmark, Finland, France, Germany, Greece, Italy, Kyrgyzstan, Lebanon, Malta, Netherlands, Poland, Portugal, Romania, Spain, Sweden, Switzerland, Ukraine, United Kingdom, former Yugoslavia.

\section{Conclusion}

With the notable exception of $C$. $u$. fuertaventurae, this review would not disagree with the general conclusion of Collar (1980) that over much of its range, the Houbara Bustard has indeed declined during the last 60 years or so. The causes of the declines appear to start with a general trend of habitat loss and degradation from agricultural intensification, overgrazing and increase in human disturbance that has affected the region and its avifauna as a whole (Goriup 1988). In some areas, this trend is compounded by intensive hunting and trapping, urbanization, oil prospecting and military activities to such an extent that local extinctions have occurred, and the distribution of the Houbara Bustard seems to be increasingly fragmented as a result.

Nevertheless, it has been shown that the Houbara Bustard remains very widely distributed (indeed its range could extend much further to the east than 
Table 2. Significant breeding season range states for the Houbara Bustard Chlamydotis undulata and population sizes either published or estimated by the author based on the evidence presented in the range state accounts

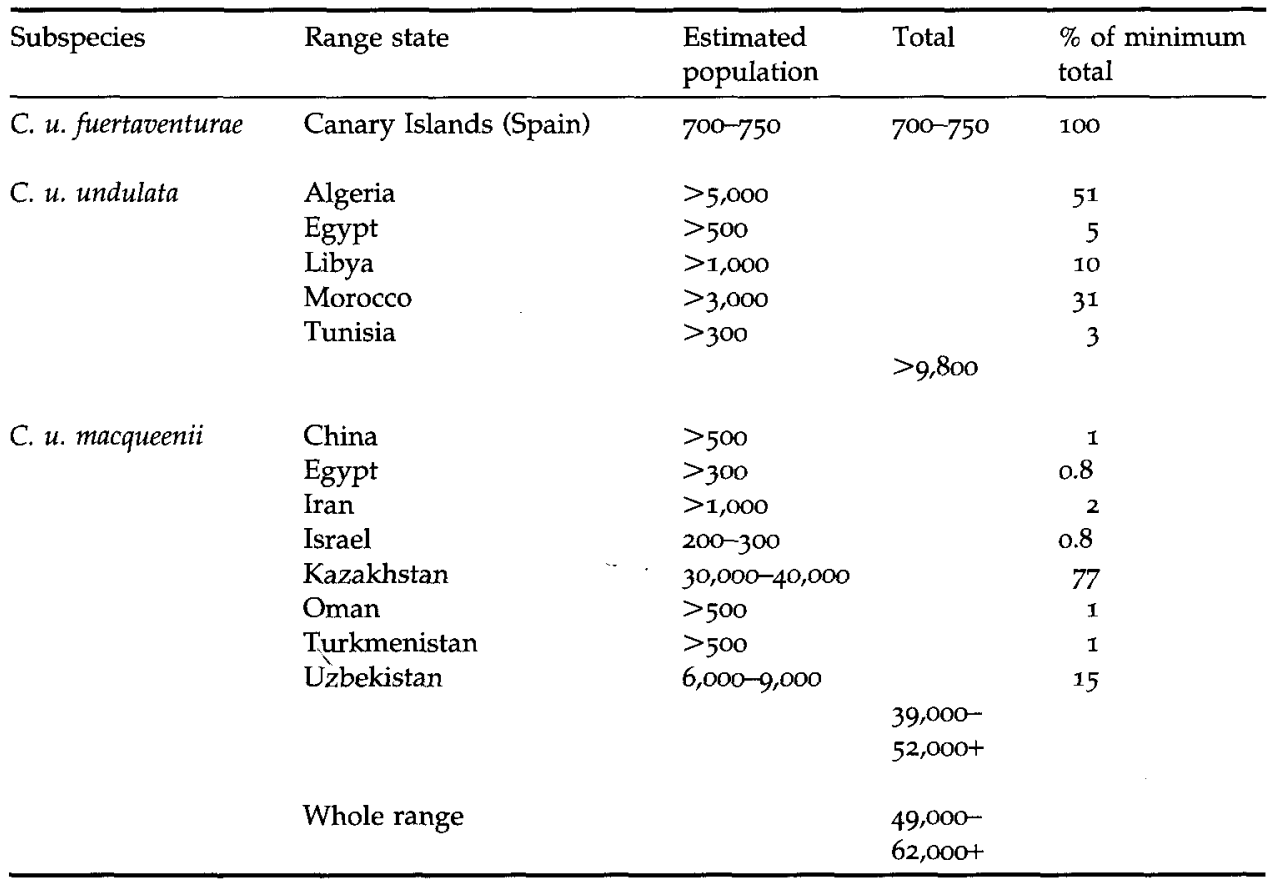

previously suspected) and, from the summary population statistics presented in Table 2, still has a relatively strong population of between 49,000 and over 62,000 birds. Furthermore, there are no good data on the rate at which declines, if any, have occurred, and whether such declines are still significant or were a feature mainly of the early and middle parts of this century. The logistic and manpower resources needed to carry out an adequate Houbara Bustard census are usually well beyond those available to ornithological fieldworkers. As Osborne (1996) has pointed out, after extensive field experience:

"Statements on Houbara abundance are at best estimates and more often informed guesswork. Censusing is made difficult by low densities, inhospitable habitats, political restrictions and seasonal movements of the birds. Houbara are particularly difficult to approach, often reacting to an observer at over $\mathrm{I} \mathrm{km}$ distance and so introducing biases into normal counting methods. When they do not move, Houbara crouch low against sand mounds or in vegetation and are very difficult to see. It is against this background that some population counts must be judged".

Yet, in the face of claims that the Houbara Bustard is facing extinction, the hunters themselves return each year to the same grounds. It does not seem likely that falconers would invest huge sums of money in hunting forays that failed to produce attractive bags.

Finally, where pressures reducing or suppressing the population are alleviated or removed, as in the Canary Islands, or in protected areas in Saudi Arabia, 
Jordan and Israel, Houbara Bustard populations can remain stable over long periods and even increase with some speed.

With the information made available in this review, and bearing in mind the above points, the level of threat posed to each of the subspecies was evaluated according to the revised criteria for classifying threatened species (IUCN 1996). The only taxon that clearly satisfied any criteria was C. $u$. fuertaventurae which meets the Vulnerable criterion $D_{1}$ of having a population of under 1,00o mature individuals. The other two subspecies probably also meet Vulnerable criterion A1 of having suffered a suspected decline of at least $20 \%$ over the past 1o years through both a contraction in range and excessive levels of exploitation. However, this criterion has been much criticized because it can include many species undergoing natural long-term population cycles or subject to sustainable harvests. It seems likely that a more stringent test of a decline of over $50 \%$ during 20 years will be adopted during the criteria review process (C. Bibby, in litt. 1997). If this is the case, then the African and Asian populations of the Houbara Bustard will remain off the Red List when it is next published in the year 2000 . Both populations (and indeed the species as a whole) can, however, be regarded as "Near threatened".

It seems clear, therefore, that the Houbara Bustard is not in immediate danger of extinction, and is not likely to be for the foreseeable future. However, it is equally clear that if harvesting for falconry (both directly by hunting and indirectly through trapping) is not conducted on a sustainable basis, then the African and Asian populations will decline more rapidly than they have done to date. The attempts by some falconers to hunt along the main migration routes and on the breeding grounds in Central Asia are particularly dangerous in this respect, and the first to suffer will be the falconers themselves as the quarry becomes too scarce to justify the time and effort to find it. It is not too late to avoid this outcome through sensible management by cooperation between the range states to protect and restore habitats, and to implement hunting policies based on sound population monitoring.

\section{Acknowledgements}

I thank colleagues who provided me with valuable insights into the recent status of the Houbara Bustard in various countries: Prof. Anatoly Kovshar (Kazakhstan), Dr Oleg Mitropolsky and Dr Elena Mukhina (Uzbekistan and Tadjikistan), Brig. Mukhtar Ahmed, Dr Abdul Aleem Chaudhry and Mr Amjad Virk (Pakistan), Dr Asad Rahmani (India), Dr Frederic Launay and Dr Patrick Osborne (U.A.E.), and Dr Brahim Haddane (Morocco). An earlier version of this paper was read at the First Meeting of the IUCN SSC Working Group on the Houbara Bustard, Muscat, Oman, 13-14 January 1996 on behalf of the UNEP-CMS (Bonn Convention) Secretariat, National Avian Research Center, Abu Dhabi, U.A.E., and IUCN-The World Conservation Union, using national reports contributed by 13 range states of the Asian Houbara Bustard involved in the Meeting Report of the IUCN SSC Working Group on the Houbara Bustard, Muscat, Oman, 13-14 January 1996. The text was amended with helpful guidance from an anonymous referee.

\section{References}

Adil, W. A. (1996) Houbara Bustard in Afghanistan. Meeting Report of the IUCN SSC Working Group on the Houbara Bustard, Muscat, Oman, 13-14 January 1996. 
Alekseev, A. F. (1980) [The Houbara Bustard in the north-west Kyzyl Kum.] Zoologicheski Zhurnal, 59: 1263-1266. [Reprinted in English in Bustard Stud. 3: 87-92, 1983]

Ali, S. and Ripley, S. D. (1969) Handbook of the birds of India and Pakistan. 2. London: Collins. Anon. (1980) Report on the Houbara Bustard. In C. L. Coles and N. J. Collar eds. Symposium Papers of the Small Game Commission of the Conseil International d la Chasse. The Great Bustard, Sofia, 1978. The Houbara Bustard, Athens, 1979. Fordingbridge, U.K.: The Game Conservancy.

Bailey, T. and Hornby, R. J. eds. (1994) National Avian Research Center Annual Report 1993. Abu Dhabi, U.A.E.: National Avian Research Center.

Bannerman, D. A. (1963) The Birds of the Atlantic Islands, 1. Edinburgh: published by author.

Baumgart, W., Kasparek, M. and Stephan, B. (1995) Die Vögel Syriens: eine Übersicht. Heidelberg: Kasparek Verlag.

Bédé, P. (1928) Notes d'ornithologie. Jardin Zoologique de Sfax, fasc. 1. Sfax.

Belgrave, J. H. D. (1953) Welcome to Bahrain. Manama and London: Augustan Press.

Blanchet, A. (1955) Les oiseaux de Tunisie. Mém. Soc. Sci. Nat., Tunisie, 3. Tunis.

Brosset, A. (1961) Écologie des oiseaux du Maroc oriental. Travaux de l'Institut Scientifique Chérifien, Serie Zoologie No. 22. Rabat.

Bulman, J. F. H. (1942) Notes on the birds of the Libyan desert. Zool. Soc. Egypt Bull. 4: 5-12.

Bundy, G. (1976) The birds of Libya. London: British Ornithologists' Union.

Chaudhry, A. A. (1996) Houbara Bustard in Pakistan. Meeting Report of the IUCN SSC Working Group on the Houbara Bustard, Muscat, Oman, 13-14 January 1996.

Clark, J. E. (1983) The Houbara Bustard in Jordan. Sandgrouse 4: 111-113.

Cloudsley-Thompson, J. L. (1992) Wildlife massacres in Sudan. Oryx 26: 202-204.

Collar, N. J. (1980) The world status of the Houbara: a preliminary review. In C. L. Coles and N. J. Collar eds. Symposium papers of the Small Game Commission of the Conseil International de la Chasse. The Great Bustard, Sofia, 1978. The Houbara Bustard, Athens, 1979. Fordingbridge, U.K.: The Game Conservancy.

Collar, N. J. and Goriup, P. D. eds. (1983) Report of the ICBP Fuerteventura Houbara Expedition, 1979. Bustard Stud. 1: 92 pp.

Collins, D. R. (1984) A study of the Canarian Houbara Bustard Chlamydotis undulata fuertaventurae with special reference to its behaviour and ecology. Unpublished MSc thesis, University of London.

Cramp, S. and Simmons, K. E. L. (1980) The Birds of the Western Palearctic, II. Oxford: Oxford University Press.

de Smet, K. (1989) The Houbara Bustard in Algeria: a preliminary report. Bustard Stud. 4: 157-159.

Dharmakumarsinhji, R. S. (1955) Birds of Saurashtra. Bhavnagar.

Dolgushin, I. A. (ed.) (1962) Ptitsi Khazakhstana, 2. Alma-Ata.

Dominguez, F. and Diaz, G. (1985) Plan de recuperacion de la Hubara Canaria. Unpublished manuscript. Madrid: Instituto Nacional para la Conservacion de la Naturaleza.

Eriksen, J. (1996). The houbara in Oman. Oman Bird News 18: 11.

Evans, M. I. (1994). Important bird areas in the Middle East. Cambridge, U.K.: BirdLife International. (Bird Conservation Series No. 2).

Felemban, H. M. and al Salamah, M. (1992) Winter counts of the Houbara Bustard Chlamydotis undulata macqueenii in Saudi Arabia. Riyadh: National Commission for Wildlife Conservation and Development.

Gallagher, M. D. and Woodcock M. W. (1980) The birds of Oman. London: Quartet.

Gaucher, P. (1988) Biological study on the reproduction of the Houbara Bustard in Algeria. Quarterly Report of the National Wildlife Research Centre, Taif. 
Gaucher, P. (1991) On the feeding ecology of the Houbara Chlamydotis undulata undulata. Alauda, 59: 120-121.

Goodman, S. M. and Meininger, P. L. eds. (1989) The birds of Egypt. Oxford: Oxford University Press.

Goriup, P. D. (1982) Houbara Bustard Chlamydotis undulata research and conservation in Pakistan. Pp. 267-272 in P. D. Goriup and H. Vardhan, eds. Bustards in decline. Jaipur: Tourism and Wildlife Society of India.

Goriup, P. D. ed. (1983) The Houbara Bustard in Morocco: report of the Al Areen/ICBP preliminary survey. Bahrain: Oriental Press.

Goriup, P. D. (1985) Bustards on the brink. Birds 10: 29-31.

Goriup, P. D. (1988) The avifauna and conservation of steppic habitats in Western Europe, North Africa and the Middle East. Pp. 145-158 in P. D. Goriup ed. Ecology and conservation of grassland birds. Cambridge, U.K.: International Council for Bird Preservation (Techn. Publ. 7).

Goriup, P. D. and Parr, D. F. (1985) Results of the ICBP bustard survey of Turkey, 1981. Bustard Stud. 2: 77-98.

Goriup, P. D., Norton, J. A. and al Salamah, M. (1992) Houbara Bustard field research project. Report for 1990. Riyadh: National Commission for Wildlife Conservation and Development.

Grimmett, R. and Taylor, H. (1992) Recent bird observations from Xinjiang Autonomous Region, China, 16 June to 5 July 1988. Forktail, 7: 139-146.

Groombridge, B. ed. (1993) I994 IUCN red list of threatened animals. Gland, Switzerland and Cambridge, U.K.: International Union for Conservation of Nature.

Gubin, B. M. (1986) [Numbers, distribution and state of protection of the Houbara Bustard in the south of Kazakhstan. Bustards in the Soviet Union.] Moscow: All-Union Ornithological Institute. [Reprinted in English in Bustard Stud. 5: 98-103.]

Heim de Balzac, H. (1924) Contributions à l'ornithologie du Sahara septontrional en Algérie et en Tunisie. Paris: Lechevalier.

Heredia, B., Rose, L. and Painter, M. (1996) Globally threatened birds in Europe. Action plans. Strasbourg: Council of Europe.

Hinz, C. and Heiss, E. M. (1989) The activity patterns of Houbara Bustards: aspects of a field study in the Canary Islands. Bustard Stud. 4: 68-79.

Hirschfeld, E. (1995) Birds in Bahrain. A study of their migration patterns 1990-1992. Dubai, U.A.E.: Hobby Publications.

IUCN (1996) IUCN red list categories. Gland, Switzerland: International Union for Conservation of Nature.

Jennings, M. C. (1995) An interim atlas of the breeding birds of Arabia. Riyadh: National Commission for Wildlife Conservation and Development.

Kroll, B. (1977) Die Scheichs und ihre Vögel. Stern no. 16: 134-140.

Kumerloeve, H. (1968) Recherches sur l'avifaune de la République Arabe Syrienne essai d'un aperçu. Alauda 36: 1-26.

Launay, F. and Loughland, R. (1995) Breeding system of Houbara Bustard Chlamydotis undulata macqueenii: preliminary observations. Bull. Orn. Soc. Middle East 35: 14-17.

Lavee, D. (1983) The influence of grazing and intensive cultivation on the population size of the Houbara Bustard in the northern Negev, Israel. Bustard Stud. 3: 103-108.

Lyster, S. (1985) International wildlife law. An analysis of international treaties concerned with the conservation of wildlife. Cambridge: Grotius.

Macfarlane, A. M. (1978) Field notes on the birds of Lebanon and Syria, 1974-1977. London: Army Birdwatching Society (Periodic Publ. No. 3).

Mann, S. (1986) Houbara survey in the Western Negev, 1986 [in Hebrew]. Unpublished manuscript. Nature Reserves Authority, Tel Aviv.

Marchant, S. (1961) Iraq bird notes: 1960. Bull. Iraq Nat. Hist. Mus. I (4). Baghdad. 
Martin, A., Nogales, M., Hernandez, M. A., Lorenzo, J. A., Medina, F. M. and Rando, J. C. (1996) Status, conservation and habitat selection of the Houbara Bustard Chlamydotis undulata fuertaventurae on Lanzarote (Canary Islands). Bird Conserv. Int. 6: 229-239.

Meinertzhagen, R. (1924) Notes on a small collection of birds made in Iraq in the winter of 1922-23. Ibis 6: 601-625.

Meinertzhagen, R. (1930) Nicoll's Birds of Egypt 2. London.

Mendelssohn, H. (1975) Report on the status of some bird species in Israel in 1974. In Barclay-Smith, P. and Chancellor, R. D, eds. Bull. Int. Council Bird Preserv. XII: 265-270.

Mendelssohn, H. (1980) Development of Houbara (Chlamydotis undulata) populations in Israel and captive breeding. Symposium Papers of the Small Game Commission of the CIC. The Great Bustard, Sofia, 1978. The Houbara Bustard, Athens, 1979. The Game Conservancy, Fordingbridge, U.K.

Mian, A. (1986) Ecological impact of Arab falconry on Houbara Bustard in Baluchistan. Environ. Conserv. 13: 41-46.

Mian, A., Shams. K. M. and Rafiq. M (1988) Biology of the Houbara Bustard (Chlamydotis undulata macqueenii) in Baluchistan. Final Technical Report (1984-1987). Unpublished. WWF-Pakistan, Lahore.

Mirza, Z. B. (1971) Houbara faces trial. Outdoorman 1: 40-42.

Mitropolsky, O. V., Mukhina, E. A. and Nazarov, O. P. (1996) Houbara Bustard Chlamydotis undulata macqueenii in Uzbekistan. Paper presented to the First Meeting of the IUCN SSC Houbara Bustard Working Group, Muscat, Oman, 13-14 January 1996.

Moore, H. J. and Boswell, C. (1956) Field Observations on the Birds of Iraq. Baghdad: Iraq Natural History Museum (Publ. No. 9).

Morel, R. (1989) A note on the status of bustards in Senegal, Mauritania and Mali. Bustard Stud. 4: 123-124.

Mukhina, A. (1989) Distribution and density of Houbara Bustard in Karnabchul (south-west Kyzyl Kum). Pp. 116-123 in Fauna and ecology of birds in Uzbekistan. Samarkand.

Newby, J. E. (1990) The slaughter of Sahelian wildlife by Arab royalty. Oryx 24: 6-8.

Nightingale, T. and Hill, M. (1993) Birds of Bahrain. London: Immel.

"Noah" (1994) Kazakhstan sponsors falcon smuggling. Ecostan News 2(12), December 1994. http://solar.rtd.utk.edu/friends/science/ecostan/ecostan.index.html

Ochaporskiy, V. S. (1986) Houbara Bustard: brief reports. Bustards in the Soviet Union. All-Union Ornithological Institute, Moscow. [Reprinted in English in Bustard Stud. 5: 105-106, 1992]

Oman Bird Records Committee (1994) Oman bird list, 4th edn. Muscat, Oman.

ORNISTUDIO (1992) Censo de la poblacion de Hubara Canaria Chlamydotis undulata fuertaventurae en la isla de Fuerteventura. Unpublished manuscript. ORNISTUDIO S. L., Santa Cruz de Tenerife.

Osborne, P. E. (1986) Survey of the birds of Fuerteventura, Canary Islands, with special reference to the status of the Canarian Houbara Bustard. Cambridge, U.K.: International Council for Bird Preservation (Study Report No. 10).

Osborne, P. E. ed. (1996) Desert ecology of Abu Dhabi: a review and recent studies. Newbury, U.K.: Pisces Publications, and Abu Dhabi, U.A.E.: National Avian Research Centre.

Paillat, P., Symens, P., Khoja, A., Boug, A. and Shobrak, M. (1988) Contribution to the biological study on the Houbara Bustard during the egg collecting expedition in Pakistan in spring 1988. Quarterly Report of the National Wildlife Research Centre, Taif.

Ponomareva, T. (1979) The Houbara Bustard: present status and conservation prospects. Okhota i okhotnich'e khozyaistvo, 11: 26-27. [Reprinted in English in Bustard Stud. 3: 93$96,1983]$ 
Ponomareva, T. (1985) Number of Houbara Bustard in Bukhara region based on aerial survey. Bull. MSRN, 90: 25-29.

Razdan, T. and Mansoori, J. (1989) A review of the bustard situation in the Islamic Republic of Iran. Bustard Stud. 4: 135-145.

Roberts, T. J. and Savage, C. D. W. (1971) Houbara Bustard Chlamydotis undulata macqueenii and its management. Outdoorman 1: 37-39.

Roshier, D. A. (1995) Anecdotal reports on the status of the Houbara Bustard Chlamydotis undulata macqueenii in Syria. Bull. Orn. Soc. Middle East 35: 18-21.

Rothschild, W. and Hartert, E. (1912) Ornithological explorations in Algeria. Nov. Zool. 18: 456-550.

Rozanov, B. G. (1977) Soviet deserts are reclaimed. Geographical Magazine, December, 162166.

Saleh, M. A. (1989) The status of the Houbara Bustard in Egypt. Bustard Stud. 4: 151-156.

Saud al Faisal (1990) Speech to the XX World Conference of the International Council for Bird Protection. Hamilton, New Zealand.

Scott, D. A. (1975) The houbara bustard in Iran. Unpublished report to the Department of the Environment, Tehran, Iran. 23 November 1975.

Seddon, P. J. (1995) Species conservation strategy for the Houbara Bustard Chlamydotis undulata macqueenii in Saudi Arabia. National Commission for Wildlife Conservation and Development, Riyadh.

Seddon, P. J. and Maloney, R. F. (1996) Reintroduction of Houbara Bustards into Central Saudi Arabia: a summary of results between 1991 and 1996. Phoenix 13: 14-16.

Seddon, P. J., Saint Jalme, M., Paillat, P., Gaucher, P. and Combreau, O. (1995) Restoration of Houbara Bustard populations in Saudi Arabia: developments and future directions. Oryx 29: 136-142.

Stanford, W. (1973) A note on the birds of Oman and the Trucial States 1954-1968. London: Army Bird-Watching Society (Periodical Publ. No. 1).

Ticehurst, C. B., Buxton, P. A. and Cheesman, R. E. (1922) Birds of Mesopotamia, part 3. J. Bombay Nat. Hist. Soc. 28: 650-674.

Tristram, H. B. (1868) On the ornithology of Palestine, 8. Ibis 4: 321-335.

Upton, R. (1989) The Houbara Bustard and the Arab falconer. Bustard Stud. 4: 174-176.

Urban, E. K., Fry, C. H., Keith, S. (1986) The birds of Africa, 2. London: Academic Press.

Valverde, J.A. (1957) Aves del Sahara Español. Madrid. Consejo Superior de Investigaciones Cientificas.

Weaver, M. A. (1992) Hunting with the Sheikhs. The New Yorker, December 14, 51-64.

Zatoka, A. (1995) Sport hunting of endangered species stopped in Turkmenistan. Anim. Welfare Int. Quarterly, Spring 1995: 13.

\section{PAUL D. GORIUP}

The Nature Conservation Bureau Ltd, 36 Kingfisher Court, Hambridge Road, Newbury RG14 5SJ, U.K. 